\title{
Distinciones y paralogismos. A propósito del escepticismo guastiniano*
}

Isabel Lifante Vidal Universidad de Alicante

\section{Introducción}

En su trabajo "El escepticismo ante las reglas replanteado", Riccardo Guastini expone muchas de las distinciones que están en la base de su "teoría" de la interpretación. En particular, empieza su trabajo analizando tres ambigüedades que afectan al término interpretación: la primera es la que distingue la interpretación en abstracto (o dirigida a textos) y la interpretación en concreto (o dirigida a casos); la segunda distingue entre la interpretación como actividad cognitiva, como actividad decisoria y como actividad creativa; y la tercera ambigüedad hace referencia a un sentido estricto de interpretación frente a un sentido más amplio que incluiría todas las actividades de construcción jurídica. El análisis de estas ambigüedades es usado por Guastini para separar aquello de lo que puede ocuparse un jurista que pretende realizar "teoría", de aquellas otras cuestiones que se adentrarían en el ámbito

* Este trabajo ha sido realizado en el marco del proyecto de investigación "Argumentación y constitucionalismo" (DER2010-21032), financiado por el Ministerio de Ciencia e Innovación español. 
de lo que Guastini considera "ideología"1 de la interpretación y con las que el teórico del Derecho debe mantener las distancias.

A partir de estos presupuestos, Guastini formula su tesis escéptica; una tesis que pretende separarse, por un lado, de la "teoría de la pesadilla" (con su escepticismo radical) y, por otro lado, de la "teoría de la vigilia" (teoría usualmente considerada intermedia entre el escepticismo y el formalismo). En opinión de Guastini, la teoría de la pesadilla está equivocada porque sostiene la tesis de la inexistencia de significado antes de la interpretación y esto atenta contra la evidencia de que existen reglas lingüísticas compartidas: "Cada atribución de significado que caiga fuera del marco de los significados admisibles no constituye propiamente una 'interpretación', sino más bien una creación de una norma nueva” (p. 53). Esta teoría escéptica radical -dice Guastini- adopta tácitamente un concepto de interpretación demasiado amplio, de manera que vuelve imposible distinguir entre comprensión y malentendido. Para Guastini la interpretación posee límites: interpretar no consiste en atribuir cualquier significado, sino en atribuir uno de los significados que se encuentran dentro del campo de significados admisibles, según a) el uso común, b) los métodos interpretativos en uso y c) las teorías dogmáticas. El error de la teoría de la vigilia consistiría, a su vez, en situar la controversia entre cognitivismo y escepticismo en el ámbito de la interpretación dirigida a hechos (enunciados subsuntivos), cuando en opinión de Guastini el objeto de interés se encuentra en los enunciados interpretativos en sentido estricto (en la interpretación dirigida a los textos). Guastini le critica el no distinguir ambos tipos de interpretación que -para él- presentan naturaleza bien distinta. Aunque consideremos -dice Guastini- que los enunciados subsuntivos pueden tener valor de verdad bajo ciertas condiciones, la cuestión relevante es si la tienen los enunciados interpretativos en sentido estricto. La teoría de la vigilia sostendría que sí; pero como teoría descriptiva ello resulta -en opinión de Guastini- bastante ingenuo, y como

\footnotetext{
${ }^{1}$ La distinción entre teoría e ideología coincide para Guastini con la distinción entre cuestiones descriptivas y cuestiones normativas. El teórico o científico del Derecho, en cuanto tal, debería en su opinión limitarse a ocuparse de las primeras.
} 
teoría normativa (que recomendaría seguir las reglas del lenguaje ordinario), se trataría de una cuestión irrelevante para una teoría descriptiva de la interpretación. El verdadero fundamento del escepticismo ante las reglas no se encuentra entonces, en opinión de Guastini, en la tesis de la inexistencia de significado previo a la interpretación, sino en "el reconocimiento de que es siempre posible interpretar un mismo texto de múltiples formas distintas, asociado con la tesis de que no existe un criterio de verdad para los enunciados interpretativos" (p. 54). Recordemos que, para Guastini, las normas siempre son variables dependientes de la interpretación ${ }^{2}$.

Su trabajo puede, por tanto, sintetizarse en dos tesis. La primera, de carácter metodológico, sostiene la posibilidad (y conveniencia) de segregar o fragmentar la experiencia jurídica en un discurso descriptivo y un discurso normativo o prescriptivo, entre los cuales existiría una distinción nítida y tajante. Me referiré a ella como la tesis del "segregacionismo discursivo" 3 . La segunda tesis, a la que denominaré tesis del "escepticismo moderado", sería una tesis sustantiva que presupone la primera pues pretende situarse exclusivamente en el ámbito del discurso descriptivo. Esta tesis se compone a su vez de dos subtesis. Por un lado, sostiene la existencia de una omnipresente indeterminación en el Derecho (toda disposición admite más de un significado) junto a la inexistencia de valor de verdad o corrección en el ámbito de la interpretación, y de ahí su carácter escéptico; pero, por otro lado, sostiene la existencia de significados previos a la interpretación que limitan la actividad interpretativa, y de ahí su carácter moderado.

${ }^{2}$ Esta tesis encuentra su fundamento en la clásica distinción genovesa entre disposición y norma. En realidad, Guastini matiza esta tesis en el sentido de indicar que el Derecho (las normas) no nacen unilateralmente de la interpretación, "sino que el Derecho nace de la combinación de dos actividades distintas igualmente "productivas", aun cuando lo sean a título diverso: la producción de textos (ésta sí, ex nihilo) y la construcción de significados" (Guastini, 2010, p. 165).

${ }^{3}$ Tomo esta terminología de Aguiló Regla, quien la utiliza para referirse a la fragmentación que realizaría Ferrajoli de la experiencia y la fenomenología jurídicas en múltiples discursos desconectados entre sí (Aguiló Regla, 2011). 
El objetivo de este trabajo es señalar mis discrepancias con esas dos tesis. Respecto a la segunda, la "tesis del escepticismo moderado", pretendo señalar el carácter inconsistente de los argumentos en que Guastini basa, por un lado, su escepticismo (la omnipresente indeterminación jurídica y la ausencia de criterios de corrección) y, por otro, su carácter moderado (la existencia de significados previos que limitan la interpretación). Intentaré mostrar que esas inconsistencias hacen imposible sostener el escepticismo moderado en los términos en que Guastini lo defiende, pues si no se abandonan los presupuestos en que fundamenta su escepticismo, el mismo acaba resultando radical (negando la existencia de significados previos); y si se quiere mantener ese carácter moderado es necesario abandonar la tesis de la omnipresente indeterminación junto a la ausencia de criterios de corrección. Para ello (en el apartado II), señalaré los problemas que presentan las distinciones guastinianas para construir una adecuada teoría de la interpretación jurídica, tomando como hilo expositivo las tres ambigüedades del término interpretación con el que inicia su trabajo. Todo ello me llevará a sostener (en el apartado III) que la primera tesis, el "segregacionismo discursivo", resulta un presupuesto metodológico inadecuado para dar cuenta de en qué consiste la interpretación en el ámbito jurídico.

\section{Las distinciones guastinianas}

Como en tantos otros temas, las distinciones guastinianas en materia de interpretación aportan una claridad y un instrumental analítico cuya utilidad resulta difícilmente exagerable. Mis discrepancias con Riccardo Guastini no tienen que ver con su aparato conceptual, sino con el uso que da al mismo para separar cuestiones y ocuparse solo de algunas de ellas presuponiendo que se pueden tratar adecuadamente de manera aislada. Intentaré mostrar que con esa estrategia no solo se dejan fuera algunas de las cuestiones más relevantes de la interpretación jurídica, sino que se genera una visión distorsionada de la misma. De este modo, mis observaciones aquí no serán tanto sobre lo que Guastini dice en su texto, sino sobre lo que calla y sobre los riesgos que ese análisis de las 
Distinciones y paralogismos. A propósito...

distinciones puede acarrear. Para presentar esos riesgos me serviré en gran medida de la figura de los paralogismos, entendiendo por tales aquel tipo de falacias que, a diferencia de los sofismas, consisten en errores involuntarios, cometidos o inducidos por confusiones a menudo inadvertidas. Seguiré en este punto el análisis que de esta figura realizó Vaz Ferreira ${ }^{4}$. Este autor considera que pertenecen a la categoría de los paralogismos, por ejemplo, las falsas oposiciones, las falsas precisiones, la extrapolación de los esquemas verbales a la realidad de las cosas o el tratamiento de las cuestiones normativas (cuyas soluciones pueden ser graduables y suelen implicar ponderaciones) como si fueran fácticas o explicativas (susceptibles de soluciones únicas y precisas). Como intentaré mostrar, el análisis de Guastini puede propiciar algunos paralogismos, especialmente falsas oposiciones, aunque conviene señalar desde ya que, tal y como sugiere Vaz Ferreira, lo que en muchas ocasiones constituye el paralogismo no es la contraposición en sí, que puede estar bien construida y resultar clarificadora en muchos contextos, sino alguna suerte de extrapolación o exceso de la misma ${ }^{5}$.

\section{Primera ambigüedad: interpretación en abstracto (o dirigida a textos) e interpretación en concreto (o dirigida a hechos)}

La primera ambigüedad que señala Guastini es la que distingue entre: (1) la "interpretación en abstracto" o dirigida a los textos y (2) la "interpretación en concreto" o dirigida a los hechos. En el primer caso se trataría de "identificar el contenido de significado -esto es, el contenido normativo (la norma o las normas)- expresado por, y/o lógicamente implícito en, un texto normativo, y ello sin hacer referencia a ningún caso concreto" (p. 28); mientras que en el segundo caso se trata

${ }^{4}$ La obra de Vaz Ferreira Lógica viva, aunque fue escrita en 1910, ha sido recogida y revisada en múltiples ediciones, de las cuales aquí cito por una de 1980. Para la presentación de las tesis de este autor he seguido muy de cerca la exposición de Vega Reñón (2008, pp. 630 y ss.).

${ }^{5}$ Cfr. Vega Reñón, 2008, p. 637. 
de "subsumir un caso concreto en el campo de aplicación de una norma previamente identificada 'en abstracto'" (p. 29). Nos encontramos, por tanto, ante dos actividades distintas: atribuir significados y clasificar casos. Aunque dichas actividades pueden darse conjuntamente -sobre todo en la interpretación judicial- y resultar desde el punto de vista psicológico como indistinguibles, Guastini remarca que desde el punto de vista lógico son realmente diferentes. En este sentido, señala que el objeto de dichas actividades sería distinto: mientras que en el primer caso se interpretarían enunciados normativos completos, la interpretación en el segundo sentido recaería "sobre predicados en sentido lógico, es decir, términos que denotan clases" (p. 30-31). Mientras que en la interpretación orientada a textos se identifican-dice Guastini- "normas jurídicas (y clases de casos)" [p. 31, la cursiva es del autor], en la dirigida a los hechos se "contribuye a identificar los casos concretos regulados por cada norma" (p. 31), por lo que -al menos desde este punto de vista lógico- la primera actividad sería anterior a la segunda. Guastini pone como ejemplo de problema de interpretación dirigida a los textos el siguiente: el artículo que establece "El Presidente de la República firma los decretos y resoluciones emanados del Consejo de Ministros" puede entenderse bien como que el Presidente "posee el poder" de firmar o bien como que "tiene el deber" de hacerlo. Mientras que, como ejemplo de interpretación dirigida a los hechos, señala la indeterminación que genera la regla "Prohibido vehículos en el parque" respecto a su aplicación o no a las bicicletas ${ }^{6}$.

${ }^{6}$ Conviene notar que este ejemplo sería un supuesto de subsunción genérica, es decir se trata de subsumir una clase de casos (o una especie), dentro de una clase más amplia (un género), pero no sería un supuesto de subsunción de un caso individual (con una determinada localización espacio-temporal) en un caso genérico (cfr. Alchourrón y Bulygin, 1987, pp. 57-61). Pese a que en la presentación de esta ambigüedad Guastini parece distinguir ambas "subsunciones", considerando que solo la primera se vincula a la interpretación "en concreto", más adelante sin embargo las trata conjuntamente, vinculando a ambas con la interpretación "en concreto" (p. 55). 
Cada uno de esos sentidos de interpretación respondería, a su vez, a un tipo de indeterminación que afecta al Derecho. La interpretación en abstracto será necesaria cuando nos encontremos ante un caso de "indeterminación del sistema jurídico como tal", es decir, cuando no están determinadas cuáles son las normas expresadas por las fuentes legales y que, en este sentido, pertenecerían al sistema; y la interpretación en concreto, o dirigida a hechos, se conectaría con lo que Guastini considera como la indeterminación de cada norma particular, que se presenta cuando no están determinados qué casos caen bajo el ámbito de aplicación de cada norma. Mientras que este segundo tipo de indeterminación depende de la vaguedad o textura abierta $^{7}$ de todo predicado del lenguaje natural, la fuente de la primera indeterminación se encuentra en la ambigüedad (en un sentido muy amplio $^{8}$ ) de los textos normativos. De este modo la indeterminación del sistema jurídico no dependerá solo de defectos objetivos del lenguaje constitucional o legislativo, sino también y fundamentalmente de la multiplicidad de métodos interpretativos, de la dogmática jurídica (que conforman una serie de presupuestos teóricos y conceptuales que, de manera inevitable, condicionan sus interpretaciones) y del sentimiento de justicia de los intérpretes (sus preferencias éticas y políticas que pueden declararse o no explícitamente).

Vinculada con esta primera ambigüedad, Guastini presenta también una distinción entre la interpretación que llevan a cabo los juristas y la interpretación que realizan los jueces. Guastini critica la poca atención que suelen prestar las que considera como teorías de la interpretación contemporáneas (la teoría del noble sueño, la teoría de la pesadilla, y la teoría de la vigilia) a la interpretación de los juristas, lo cual constituye en su opinión un grave defecto porque, en primer lugar, ambas actividades no serían equivalentes desde el punto de vista del

${ }^{7}$ Guastini considera que no es necesario distinguir aquí entre estos dos fenómenos (p. 39), y yo tampoco lo haré.

${ }^{8}$ Guastini incluye en este sentido de ambigüedad -no exclusivamente sintácticotodos aquellos casos en los que consideramos que una disposición puede ser entendida de diversos modos, y por tanto se duda de qué normas expresa. 
análisis lógico (así, por ejemplo, señala que la interpretación judicial nunca puede ser meramente cognitiva ni dirigida a textos) y, en segundo lugar, porque la actividad de los juristas (sus teorías dogmáticas y sus métodos interpretativos) condiciona "las ideas que los jueces poseen acerca del derecho en general, de los conceptos jurídicos y de la interpretación judicial" (p. 45). De hecho, para Guastini estas actividades de los juristas dogmáticos ayudan a conformar el marco de significados admisibles para un texto normativo. Esta crítica está conectada con una observación más general que recorre todo el trabajo: la denuncia de que las teorías de la interpretación se hayan centrado en el problema de la vaguedad, y por tanto en la interpretación en concreto, descuidando lo que sería la interpretación en abstracto. En este sentido, Guastini parece sugerir que deberíamos reservar el término "interpretación" para la interpretación en abstracto; mientras que la segunda, la interpretación en concreto, sería -o mejor dicho, incorporaría- una actividad posterior a la interpretación, consistente en subsumir casos particulares en las normas previamente interpretadas. Pero en realidad no todas sus apreciaciones resultan coherentes con este presupuesto y en ocasiones parece, por así decir, "ontologizar" la distinción, dando a entender que hay casos que "son" (no solo que se presentan como) problemas de interpretación en abstracto y casos que "son" problemas de interpretación en concreto. Y así, por ejemplo, sostiene que la interpretación en abstracto estaría dirigida a resolver los problemas originados por la indeterminación del sistema jurídico (la ambigüedad en sentido amplio), mientras que la interpretación en concreto trataría de superar la indeterminación de las normas particulares, es decir, la generada por la vaguedad.

Todo ello parece dar a entender que no estamos simplemente ante dos sentidos del término "interpretación" uno de los cuales incluye, además de lo que sería en sentido estricto actividad interpretativa (la encargada de eliminar la indeterminación del significado de la disposición), una posterior actividad subsuntiva o calificativa, ni tampoco ante dos perspectivas desde las que pueda plantearse la 
necesidad de llevar a cabo una misma actividad interpretativa ${ }^{9}$. Esta manera de presentar las cosas, remarcando las diferencias entre los dos "tipos" de interpretación, puede generar algunas distorsiones, llegando a generar incluso un paralogismo: me refiero a sugerir lo que sería una falsa oposición entre ambas actividades.

La cuestión de si las bicicletas son o no vehículos a efectos de la regla "Prohibida la entrada de vehículos en el parque" sería un problema de vaguedad del concepto de "vehículo", pero hace referencia a la subsunción de un caso genérico, de una clase (la de las bicicletas) en otra clase (los vehículos), y no a la subsunción de un caso concreto o individual, por lo que no se acaba de entender por qué Guastini lo pone como ejemplo de "interpretación en concreto". Pero incluso si se tratara de determinar si la prohibición se aplica a una bicicleta particular en un caso concreto (identificable en el tiempo y el espacio), el modo de resolver dicha indeterminación generada por la vaguedad habría consistido en "construir" un caso genérico con las propiedades que se consideran relevantes del caso individual (tener dos ruedas, no tener motor...). Por lo tanto, si consideramos aplicable la regla "Prohibida la entrada de vehículos en el parque" a las bicicletas es porque hemos interpretado la regla en una de sus opciones (no solo hemos calificado un caso concreto). Si bien es cierto que la subsunción de un caso particular en una regla, una vez atribuida a ésta un significado preciso (una vez resueltas las posibles indeterminaciones que su formulación pueda plantear), es una mera cuestión lógica, la resolución de la indeterminación provocada por la vaguedad o por la textura abierta de algunos de los conceptos usados en la formulación de la regla será siempre una actividad interpretativa en sentido estricto.

Pensemos en otro ejemplo. El artículo 85 del Estatuto de la Universidad de Alicante establece lo siguiente: "El mandato de decano

${ }^{9}$ Este sería el modo en el que, por ejemplo, Ross plantearía sus dos "formas" de la interpretación: la interpretación por significado y la interpretación por resultado (Ross, 1970, p. 113) o también MacCormick su distinción entre los problemas de interpretación y de calificación (MacCormick, 1978, pp. 94 y ss.), distinciones que coincidirían en gran medida con la que aquí estamos analizando. 
tendrá una duración de 4 años, siendo posible su reelección una sola vez". Nos encontramos con una indeterminación que puede ser planteada como un problema de "interpretación en abstracto" generado por la indeterminación del sistema jurídico: ¿Qué norma expresa esta disposición?: N1, según la cual se estarían limitando las elecciones consecutivas de los decanos, o N2, según la cual se limitarían el número de mandatos que en total puede desempeñar un decano. Pero que también puede plantearse como problema de "interpretación en concreto": ¿Es un caso de "reelección" elegir a Fulanito que, en el pasado, fue decano durante dos mandatos? Cualquiera de los problemas de "interpretación en concreto" podría surgir o presentarse como un problema de interpretación en abstracto y es importante darse cuenta de que la "actividad interpretativa" necesaria para superar la indeterminación sería la misma en ambos casos. Como el propio Guastini remarca, la diferencia entre los dos sentidos se encuentra en que en la llamada "interpretación en concreto" se incluiría algo más, que vendría a ser precisamente la subsunción de un caso particular en la regla interpretada, pero eso implica considerar que existe un núcleo de actividad común.

En realidad, en la caracterización que hace Guastini de la llamada "interpretación en concreto" no se parte solo de un caso -ya sea genérico o individual- frente al cual busquemos una solución en "el Derecho" (considerado en su conjunto), sino que se tiene identificada previamente una disposición que expresa una norma respecto a la que se plantea la duda de si el caso resulta o no subsumible. Esta ambigüedad -tal y como es presentada por Guastini- hace referencia, por tanto, a dos sentidos de "interpretación" en los que la actividad interpretativa se realiza siempre a partir de una determinada disposición cuyo significado nos plantea dudas con un mayor o menor nivel de abstracción (generados bien por ambigüedad, bien por vaguedad), y en este sentido podemos decir que la "interpretación en concreto o dirigida a hechos" estaría también dirigida a textos. Por decirlo de otro modo, para Guastini el significado central del término interpretación coincide con lo que Tarello (1980, pp. 5 y ss.) considera el sentido jurídico moderno de dicha expresión (lo que sería la "interpretación de la ley" frente a la "interpretatio iuris"), y que 
Distinciones y paralogismos. A propósito...

dejaría fuera el problema de encontrar en el Derecho como un todo la regulación jurídica aplicable, la norma que dé solución a un caso concreto. Como veremos al analizar la tercera ambigüedad, este tipo de actividad se ubicaría no en la interpretación en sentido estricto, sino en la construcción jurídica.

\section{Segunda ambigüedad: interpretación cognitiva, inter- pretación decisoria e interpretación creativa}

La segunda ambigüedad -inspirada en Kelsen- muestra que usamos el término "interpretación" para hacer referencia a actividades de naturaleza bien distinta. Nos encontramos así, según Guastini, con tres posibilidades: (1) una "interpretación cognitiva" que consistiría en "identificar, sin elegir entre ellos, los posibles y diferentes significados de un texto normativo (los significados admisibles según las reglas lingüísticas -sintácticas, semánticas y pragmáticas- compartidas, las distintas técnicas interpretativas en uso, y las tesis dogmáticas difundidas en doctrina)"; (2) una "interpretación decisoria" que consistiría en "elegir un determinado significado entre los significados identificados (o identificables) por medio de la interpretación cognitiva, descartando los restantes"; y (3) una "interpretación creativa" que consistiría en "atribuir a un texto un significado 'nuevo' no comprendido entre los significados identificados (o identificables) por medio de la interpretación cognitiva" (pp. 31-32). Siguiendo también a Kelsen, Guastini señala que mientras la primera operación está privada de cualquier efecto práctico, la segunda y la tercera serían sin embargo operaciones políticas que pueden ser llevadas a cabo tanto por órganos de aplicación (sería el caso de lo que Kelsen calificaba como interpretación auténtica, que estaría dotada de consecuencia jurídicas) como por juristas (en cuyo caso no posee dichas consecuencias). Respecto a esta ambigüedad, Guastini señala que la tercera operación, la interpretación creativa, en la mayoría de los casos consiste en extraer del texto normas no expresas mediante una amplia variedad de argumentos "no deductivos". Dicha operación no sería estrictamente hablando un acto de "interpretación", sino que estaría más próxima a la creación o legislación de nuevas normas y 
considera que esta actividad se incardinaría en lo que considera como "construcción" o integración del Derecho; lo que le lleva a presentar la tercera ambigüedad.

Me centraré primero en la distinción entre las dos primeras categorías, que son las que integrarían lo que Guastini considera como "interpretación en sentido estricto", para pasar luego a la contraposición que hace entre ellas y la "interpretación creativa". Lo que aquí pretendo mostrar es que, pese a que aparentemente el criterio de la distinción entre estos tres sentidos de interpretación radica en el tipo de actividad que se lleva a cabo en cada caso, Guastini en realidad elude abordar la cuestión a propósito de en qué consiste la actividad interpretativa.

Según su presentación, la interpretación "cognitiva" parece consistir en identificar, sin elegir entre ellos, los posibles significados de un texto normativo, mientras que la interpretación "decisoria" consistiría en elegir un determinado significado entre los significados identificados (o identificables) por medio de la interpretación cognitiva. Solo la segunda actividad tiene, en opinión de Guastini, alcance práctico; siendo la primera una actividad puramente cognoscitiva o teórica. La interpretación cognitiva parece ser entendida entonces como una mera recolección de las distintas interpretaciones resultado (los posibles significados de una disposición normativa). Pero si utilizamos aquí una distinción que en muchas ocasiones ha realizado el propio Guastini entre lo que sería un discurso "interpretativo" y un "discurso descriptivo de interpretaciones (el discurso que constata o refiere, a nivel de metalenguaje, que, de hecho, un texto ha sido interpretado de una determinada forma por parte de alguien)" (Guastini, 1999, p. 205), podríamos decir que en realidad aquí no estaríamos tanto ante una actividad propiamente interpretativa, sino ante la referencia a las distintas interpretaciones realizadas por $\operatorname{otros}^{10}$. Y, en este sentido,

10 También Barberis lleva a cabo un comentario similar, señalando que esta interpretación cognitiva, que Guastini suele considerar asimilada a la definición lexicográfica, indica en realidad no interpretación, sino descubrimiento [rilevazione] de la interpretación realizada por otros (Barberis, 2000, pp. 21-22). 
podría ser considerada -tal como hace Guastini- como una actividad meramente descriptiva, cuyo resultado puede ser calificado como verdadero o falso; pero creo que nadie -ni siquiera el propio Guastinidiría que en eso consiste la actividad de "interpretar": sería una actividad "cognitiva", pero no "interpretativa".

Sin embargo, la caracterización que Guastini realiza de la "interpretación cognitiva" admite también otra lectura, pues parece que esta interpretación debe señalar tanto los significados "atribuidos" a una disposición, como también los significados "atribuibles". Si esto es así, debemos considerar que en la categoría de la interpretación cognitiva se incluyen dos actividades de alcance muy distinto. Señalar los significados atribuidos (por otros juristas teóricos o prácticos) a una disposición puede ser considerado como una actividad meramente cognoscitiva, carente de relevancia práctica. Pero eso mismo ya no es tan obvio respecto a la identificación de los significados "atribuibles", es decir, significados que todavía no han sido atribuidos por nadie, pero que podrían ser atribuidos, es de suponer atendiendo a algunas normas o pautas que regulan dicha actividad (recordemos que para Guastini no todo vale en materia de interpretación: no puede ser atribuido ${ }^{11}$ cualquier significado imaginable, sino que existen límites marcados por las reglas lingüísticas, los métodos interpretativos y las construcciones dogmáticas). Respecto a estos últimos significados (los atribuibles pero aún no atribuidos) sería necesario llevar a cabo una actividad para determinar su "atribuibilidad", actividad que ya no puede ser considerada descriptiva de las interpretaciones-producto realizadas por otros, y en este sentido no puede limitarse a observar la práctica interpretativa, sino que tendrá que participar en ella en el sentido de que deberá aplicar esas pautas o criterios que son las que dan lugar a los distintos significados "atribuibles". Si no me equivoco, el producto de esta actividad interpretativa se incardinaría en los enunciados

${ }^{11}$ Es cierto que este "no puede ser atribuido" no pretende tener para Guastini un alcance "normativo" (no pretende guiar la práctica jurídica), sino meramente "conceptual": si se traspasaran esos límites, entonces -sostendría Guastini- eso ya no sería interpretación. 
interpretativos que Chiassoni considera como "conjeturales"12: los que "enumeran algunos -o, al límite, todos- los significados posibles y/o razonables de una disposición a la luz de los diversos métodos interpretativos culturalmente aceptados y/o positivamente prescritos" (Chiassoni, 1998, p. 23). Se trata de enunciados -dice Chiassoni- que "documentan una actividad interpretativa que versa inmediatamente no ya sobre los discursos interpretativos de disposiciones, sino sobre las propias disposiciones" (ibid. p. 26), y en ese sentido se diferenciarían de los enunciados que ponen de manifiesto (o predicen) interpretacionesproducto de otros, pero -en su opinión- al igual que éstos presentarían una función cognoscitiva o informativa, que los diferenciaría de los diversos tipos de enunciados interpretativos que considera "adscriptivos" de significados. Sin embargo, la "actividad que documentan" estos enunciados conjeturales puede exigir la aplicación de métodos interpretativos culturalmente aceptados, tales como el "método sistemático", que -al menos en alguna de sus versiones- exige la coherencia del sentido atribuible a una disposición con los fines y valores que se pretenden desarrollar en el ámbito jurídico en cuestión. Y parece que la actividad necesaria para determinar dicha coherencia ya no es tan claramente una actividad meramente cognoscitiva y carente de relevancia práctica. Y lo mismo podría decirse de la actividad necesaria para considerar atribuible un significado a la luz de una determinada construcción dogmática. En realidad, las actividades de este tipo (determinar la coherencia de un posible significado de una disposición con el resto del sistema, o con una construcción dogmática) estarían más próximas a lo que usualmente consideramos como actividad interpretativa, que la mera referencia a las interpretaciones realizadas por otros, pero respecto a su naturaleza Guastini no se pronuncia.

Por lo que respecta a la categoría de la interpretación decisoria, en este caso la actividad interpretativa es presentada por Guastini como la elección de una de las diferentes opciones previamente identificadas (o

12 Chiassoni realiza una enumeración de hasta diez diferentes tipos de enunciados que se incardinarían en la clase que generalmente se considera como "enunciados interpretativos" en sentido amplio (Chiassoni, 1998). 
identificables) a través de la interpretación cognitiva. Pero, ¿qué sentido tiene decir que la naturaleza de la actividad interpretativa es "decisoria" o electiva? Lo típicamente interpretativo no es la "decisión" en sí, sino precisamente la actividad previa que conduce a la elección de una de las diferentes opciones. La "decisión" sería la última fase, el resultado de un proceso, pero para llegar ahí ha sido necesario realizar una cierta actividad previa a la toma de decisión, una actividad consistente en deliberar, en ponderar razones. La actividad de "interpretar" acabaría con un momento decisorio ("interpretar X en el sentido Y"), pero interpretar no consiste solo -ni fundamentalmente- en decidir, sino en llevar a cabo la actividad que conduce a la decisión. De este modo, decir que la actividad interpretativa, cuando no es meramente cognitiva, consiste en "decidir", parece obviar la cuestión relevante: ¿cómo se decide?, ¿qué tipo de razones se admiten para justificar la elección en este ámbito concreto? Como hemos visto, Guastini admitiría la existencia de una multiplicidad de métodos, instrumentos o criterios interpretativos, pero no la existencia de criterios (o si se quiere, "metacriterios") que permitan comparar los distintos resultados a los que dichos métodos conducen y que en ese sentido rijan la toma de decisión: la elección de uno de esos resultados. De modo que estos criterios, al no existir, no pueden ser descritos y por tanto no tiene sentido que sean objeto de una "teoría" de la interpretación. Ocuparse de tales criterios implicaría -en su opinión- adentrarse en el ámbito de la ideología.

Si pasamos ahora a la distinción entre la interpretación en sentido estricto y la tercera categoría, la de la interpretación creativa, nos encontramos con una cierta circularidad. Lo que hace que algo sea considerado como interpretación en sentido estricto es precisamente el que el significado elegido se encuentre dentro de las opciones previamente identificadas como atribuibles a la disposición. Es decir, se supone que es la interpretación cognitiva la que marca el ámbito de los significados aceptables (atribuibles) y entre los que se puede optar a la hora de llevar a cabo la interpretación decisoria. Pero, a su vez, en la construcción escéptica de Guastini el único criterio cierto para determinar cuáles son todos los significados que han de ser reconocidos 
como atribuibles a una disposición y por tanto incorporados en el ámbito de la interpretación cognitiva parece ser, en último término, el hecho de que los juristas (teóricos o prácticos) decidan darle ese significado a la disposición. Recordemos que, en su opinión, el sentimiento de justicia de los juristas o sus construcciones dogmáticas (ambos considerados por él como "arbitrarias") pueden hacernos considerar como aceptable un significado que se aparte incluso del significado marcado por el uso común, y si ese sentimiento de justicia o esas construcciones dogmáticas son caracterizadas por el propio Guastini de arbitrarias, difícilmente podrán ser objeto de predicción antes de que se lleven a cabo. De modo que, en última instancia, acabaría siendo la interpretación decisoria la que determina el abanico de significados que se deben incorporar en la interpretación cognitiva (y no al contrario, como parece presuponer la construcción de Guastini). Ello tendría además como consecuencia inevitable que se desvanecería la distinción entre la actividad interpretativa en sentido estricto y la actividad creativa, tal como la formula Guastini. Pues si no tenemos identificados todos los criterios para determinar ex ante cuáles son los significados que pueden ser atribuidos, no hay manera de distinguir entre la interpretación y la pura creación. Y esa distinción resulta central para poder seguir sosteniendo la tesis del "escepticismo moderado"; si esa distinción se diluye acabaríamos cayendo en la tesis de que todo cabe en materia de interpretación, es decir, en el escepticismo radical del que Guastini pretende apartarse ${ }^{13}$. Es cierto que aquí podría señalarse que juega un factor temporal: son los resultados de las interpretaciones decisorias del pasado (o al menos los que han tenido cierto éxito) los que deben integrarse en el marco de posibilidades de la interpretación cognitiva del presente. Pero esto implicaría sostener que toda interpretación que es atribuida por primera vez a un texto, y que no se limite a ser una interpretación literal (o aplicación de algún método interpretativo claro y unívoco -si lo hubiera), no sería en sentido estricto "interpretación", sino pura creación, pero esto parece apartarse mucho del uso común del término "interpretación". La explicación de estas

${ }^{13}$ Cfr., en este mismo sentido, Barberis, 2000, pp. 33 y ss. 
incongruencias sigue estando -en mi opinión- en una deficiente caracterización de la naturaleza de la actividad interpretativa, lo que nos lleva al análisis de la tercera y última ambigüedad. Como intentaré mostrar en el resto del trabajo, sólo a partir de una caracterización de la interpretación como una actividad argumentativa inserta en una práctica en la que operan criterios evaluativos de corrección puede romperse esa circularidad que amenaza a la tesis de la existencia de límites a la actividad interpretativa.

\section{Tercera ambigüedad: interpretación y construcción jurídica}

Con el vocablo "interpretación", señala Guastini, se hace referencia en ocasiones al conjunto de todas las operaciones de los juristas y los jueces. Pero entre ellas existen diferencias importantes. En particular, Guastini considera necesario distinguir entre (1) la "interpretación propiamente dicha" y (2) la "construcción jurídica"; es decir, entre la atribución de significado a textos normativos (en alguno de los sentidos y modos antes señalados) y un sentido más amplio que abarcaría las numerosas operaciones en las que por lo general consiste el trabajo de juristas y jueces, y que incluiría cosas tales como "conjeturas acerca de la denominada ratio legis, las hipótesis contrafácticas acerca de la intención del legislador, la creación de jerarquías axiológicas entre normas, la construcción de reglas no expresas, la concreción de principios abstractos, la ponderación entre principios (especialmente constitucionales) en conflicto, etc." (p. 34). Entre todas estas operaciones, Guastini destaca la construcción de normas no expresas, es decir, normas que "no pueden ser consideradas como uno de los contenidos de significado o como la consecuencia directa de una determinada disposición". Toda norma no expresa -continúa Guastinies el resultado de un razonamiento en el que alguna de sus premisas es una norma expresa. Pero llama la atención sobre el hecho de que en la mayoría de los casos tales argumentos no son lógicamente válidos y además señala que "incluyen premisas que no son reglas expresas, sino esquemas conceptuales y teorías elaboradas arbitrariamente por la dogmática" (la cursiva es mía). De este modo la "interpretación creativa" excedería los límites de lo que en su opinión debe considerarse 
como interpretación en sentido estricto al necesitar de este tipo de actividad constructiva para formular el significado que se atribuiría a una disposición.

Esta distinción entre "interpretación" y "construcción -o integraciónjurídica" puede tomarse como una ambigüedad de la expresión "interpretación", frente a la cual no habría mucho que objetar. Creo que es obvio que en ocasiones hablamos de "interpretación" en un sentido más restringido, que parte siempre de una disposición (o fragmentos de disposiciones) sobre cuyo significado se duda; y sin embargo en otras ocasiones (cuando por ejemplo hablamos de "interpretación del Derecho" frente a "interpretación de la ley"14) incluimos en ese rótulo todas las actividades que se integrarían en el denominado "método jurídico". Pero el riesgo de la presentación de Guastini se encuentra en que genera la apariencia de que nos encontramos ante actividades no solo distintas, sino también incompatibles: si algo es integración, o construcción -parece sostener Guastini-, entonces no es interpretación. La interpretación en sentido estricto de la que habla Guastini parece excluir, por definición, las actividades de construcción jurídica. Si ello es así, nos encontramos de nuevo ante un paralogismo generado por una falsa oposición entre la "interpretación" y la "construcción jurídica", y ello porque aunque es cierto que no todas las actividades que se integran en la llamada construcción jurídica serían realizadas con el objetivo de llevar a cabo una interpretación, la actividad interpretativa requiere realizar, si no siempre al menos en la gran mayoría de las ocasiones, el tipo de actividades que Guastini consideraría como construcción jurídica ${ }^{15}$. Son precisamente esas actividades que aquí se incluyen (ponderación de principios, jerarquización de valores, construcciones sistemáticas, etc.), y a las que Guastini deja fuera del objeto de estudio de la teoría de la interpretación jurídica, las que resultan necesarias para conducir a las propuestas de significados que se considerarían

${ }^{14}$ Siguiendo en este punto a Tarello (1980, pp. 5-11), en ocasiones anteriores me he ocupado de caracterizar estos dos sentidos de "interpretación" (Lifante Vidal, 2010, pp. 45-50).

${ }^{15}$ En un sentido similar, Barberis también señala que Guastini no consigue distinguir adecuadamente entre la interpretación en sentido estricto y la integración del Derecho (Barberis, 2000, p. 22). 
"atribuibles" a una disposición y que se integrarían por tanto en la interpretación cognitiva y también serían este tipo de actividades las que se requieren para evaluar y comparar entre sí los distintos significados prima facie atribuibles a una disposición antes de elegir uno de ellos (interpretación decisoria). De este modo, creo que la cuestión a propósito de en qué consiste la actividad interpretativa que estaba eludida en el análisis de Guastini requiere tomar en cuenta conjuntamente diversos sentidos señalados por Guastini. Si nos ocupamos de la actividad interpretativa (y no solo de su resultado), la interpretación ha de verse como un proceso que pasa por distintas fases. Mientras que la primera fase se asemejaría bastante a lo que Guastini considera como una actividad cognitiva, la última requeriría la toma de una decisión. Pero entre ellas necesitamos una actividad de construcción, sistematización de las normas jurídicas, ponderación de principios, resolución de antinomias, etc. Si bien se mira, estas distintas fases se asemejan bastante a las diversas etapas interpretativas de las que habla Dworkin ${ }^{16}$.

Guastini señala que estas actividades constructivas o de integración que llevan a cabo los juristas exceden del ámbito de la lógica deductiva, pero el problema es que de esa tesis (que sería difícilmente refutable) parece dar el salto a presentarlas como una cuestión de mera arbitrariedad (la construcción sería entonces una invención), dando a entender que no hay espacio para la racionalidad entre ambos extremos: todo lo que no caiga dentro del ámbito de la lógica deductiva -parece suponer- no tiene más remedio que ubicarse en el terreno de la arbitrariedad. Se trataría de nuevo de un paralogismo generado por una falsa oposición, en particular del tipo que Vaz Ferreira considera como su modalidad más extendida: tomar por opuestos contradictorios dos extremos (la racionalidad deductiva y la arbitrariedad) que más bien serían contrarios. En mi opinión podríamos decir que la "naturaleza" de

${ }^{16}$ Dworkin, 1986, pp. 65 y ss. Aunque Dworkin no es muy claro al respecto, creo que la mejor comprensión de dichas fases o etapas pasa por verlas no como integrantes de un proceso estrictamente temporal, sino considerar que entre ellas se daría, por decirlo con Dworkin, una relación parecida al equilibrio reflexivo rawlsiano. 
la actividad interpretativa es fundamentalmente "constructiva", en el sentido de que no es meramente cognitiva (no se trata solo de describir algo que preexiste), pero tampoco puede reducirse a una mera decisión arbitraria (que "inventaría" un significado). Ello, aunque no implica comprometerse con la tesis de la existencia de una única respuesta correcta para todos los problemas interpretativos, sí requiere -como veremos- sostener una visión no escéptica de la racionalidad práctica.

\section{La reconstrucción de la práctica interpretativa (entre la descripción y la prescripción)}

Como hemos visto, frente al escepticismo radical, Guastini pretende sostener un "escepticismo moderado" que aceptaría la existencia de significados previos que determinarían los límites de la actividad interpretativa, pero que al mismo tiempo negaría la existencia de criterios normativos que permitan evaluar la corrección/incorrección de las distintas interpretaciones. Respecto a esta última cuestión Guastini considera que no hay nada malo en las teorías normativas del significado jurídico (como la teoría del significado literal o la teoría intencionalista del significado), "salvo que ellas no son de ayuda para describir qué es en realidad la interpretación"; ocuparse de tales criterios normativos implicaría adentrarse en el ámbito del discurso prescriptivo o de la ideología que debe quedar fuera de una teoría de la interpretación jurídica. Sin embargo, tal y como hemos visto, esos dos componentes de la tesis del "escepticismo moderado" (la existencia de significados previos y la omnipresente indeterminación junto a la ausencia de criterios de corrección) entran en conflicto, haciendo prácticamente imposible sostener ese pretendido escepticismo moderado, además de no poder caracterizar adecuadamente en qué consiste la actividad interpretativa. Lo que ahora pretendo mostrar es que el origen de esos problemas quizás se encuentre precisamente en ese presupuesto metodológico, al que he denominado la tesis del "segregacionismo discursivo", que exige la nítida separación entre el discurso teórico/descriptivo y el práctico/normativo.

Según Guastini, los significados preexistentes que limitan la actividad interpretativa, señalando el marco de posibilidades interpretativas 
atribuibles a una disposición vienen configurados por la propia actividad interpretativa llevada a cabo por la dogmática y los juristas en general. De modo que la caracterización de lo que sería propiamente interpretación, como algo distinto a la pura creación o invención, exige prestar atención a la práctica interpretativa de los juristas. En este sentido, cuando Guastini señala que en el ámbito jurídico no tiene sentido hablar de una teoría del significado como algo distinto a una teoría de la interpretación, afirma: “¿En qué puede consistir una teoría puramente descriptiva del significado sino en la reconstrucción de la práctica efectiva de una determinada comunidad interpretativa? La teoría del significado referida a textos jurídicos no puede ser otra cosa más que el análisis y la reconstrucción racional de la práctica de atribución de significado llevada a cabo por los intérpretes jurídicos" (p. 50). Ahora bien, si la teoría del significado, o teoría de la interpretación jurídica, ha de ser una reconstrucción racional de la práctica, hemos de tomarnos en serio en qué consiste precisamente dicha práctica y qué implica su reconstrucción.

Para empezar, la teoría de la interpretación tendría que dar cuenta de que la actividad interpretativa es fundamentalmente una actividad constructiva que implica llevar a cabo una argumentación, no es por tanto un mero acto de conocimiento ni tampoco un mero acto de voluntad, aunque contiene algo de ambos en las diversas etapas o momentos del proceso interpretativo. Suele considerarse que la forma estándar de un enunciado interpretativo en el ámbito jurídico, lo que sería el resultado de ese proceso interpretativo, sería la siguiente: "D significa N", pero es importante darse cuenta de que dicho enunciado, al ser el resultado de una actividad constructiva, sería la conclusión de una argumentación (práctica, no meramente teórica) en la que se dan razones para preferir el significado "N" a otros posibles. Ese enunciado interpretativo (cuando es usado en un discurso interpretativo, y no en un discurso descriptivo de las interpretaciones realizadas por otro ${ }^{17}$ ) vendría a ser equivalente a un enunciado del tipo " $\mathrm{D}$ debe ser entendido como

${ }^{17}$ Como antes hemos visto, esta distinción de los dos usos de los enunciados interpretativos es señalada por el propio Guastini (1999, p. 205). 
$\mathrm{N}$ ", o "Es mejor entender $\mathrm{D}$ en el sentido $\mathrm{N}$ que en cualquier otro sentido", en los que encontramos necesariamente una pretensión de corrección. Del mismo modo que aceptamos que un enunciado descriptivo del tipo "El gato está sobre el felpudo" incorpora la pretensión de verdad de dicha aserción, tenemos que estar dispuestos a aceptar que un enunciado interpretativo del tipo "D significa $\mathrm{N}$ " incorpora una pretensión de corrección. En ambos casos las pretensiones apelan a los otros, son en este sentido intersubjetivas. Obviamente los criterios compartidos que operan en el caso de una y otra pretensión no son los mismos. En el caso de la pretensión de verdad los criterios compartidos apelan a la idea de verificabilidad empírica, mientras que en el caso de la pretensión de corrección se apela a otro tipo de criterios como puede ser la aceptabilidad pragmática a la luz de los fines y valores que pretende desarrollar la práctica en cuestión. Necesitamos por tanto criterios válidos intersubjetivamente, criterios que no pueden reducirse a mera subjetividad ni arbitrariedad.

Una práctica social como puede ser la práctica interpretativa jurídica viene constituida por una serie de comportamientos regulares observables empíricamente, pero no solo -ni quizás fundamentalmentepor eso. Cualquier práctica ${ }^{18}$ incorpora una serie de valores que pretenden desarrollarse a través de la misma y que serían los que darían lugar al aspecto normativo de la práctica y se utilizarían como criterios para valorar la preferencia de unas soluciones sobre otras para los

${ }^{18}$ En filosofía de la ciencia, Rouse (2008) defiende que no sólo en el ámbito de las prácticas lingüísticas o argumentativas debe operar una concepción normativa de "práctica" (como opuesta a la concepción de las prácticas como regularidades), sino que dicha concepción es también la adecuada para dar cuenta de las prácticas científicas. En este mismo sentido, Sergio Martínez ha remarcado la idea de que ninguna práctica científica puede ser caracterizada si nos desentendemos de su "estructura normativa", en la que se integrarían valores de diferente naturaleza (entre otros, morales y epistémicos). Son esos valores y normas que forman parte de la práctica los que permiten dar cuenta de la estabilización y la integración de la práctica en un contexto normativo más amplio y los que permiten hablar de los "fines" de la práctica (Martínez, 2008, p. 160). 
problemas que se planteen al seguir la práctica. No se trata, por tanto, de que la teoría de la interpretación deba prescribir cómo interpretar al margen de cómo se lleva a cabo dicha actividad en la práctica, sino que de lo que se trata es de percatarse de que la interpretación es una actividad normativa que incorpora criterios de corrección, y como tal ha de ser reconstruida. Una de las cuestiones relevantes que la teoría de la interpretación ha de abordar será entonces la reconstrucción de dichos criterios de corrección.

Guastini solo analiza dos posibles candidatas a dar cuenta de la normatividad o de los criterios de corrección de la interpretación jurídica: la teoría del significado literal y la teoría intencionalista; aunque, fiel a su presupuesto metodológico, considera que las mismas pueden interpretarse bien como teorías puramente descriptivas (en cuyo caso -dice Guastini- resultarían descripciones falsas de las conductas constitutivas de la práctica), o bien como teorías prescriptivas que pretenderían guiar -y, podríamos añadir, cambiar- la práctica, y sobre cuya "conveniencia" Guastini no se pronuncia ${ }^{19}$. Pero detengámonos un momento en ellas. Según la primera teoría, la interpretación jurídica debería ser acorde con el significado literal de las palabras usadas en los textos normativos (o, por decirlo de otro modo, una interpretación sería correcta cuando así lo hiciera), mientras que, para la segunda teoría, la interpretación jurídica debería ser acorde con la intención del legislador. Si nos fijamos bien, en ambos casos se trabaja con un criterio de corrección que puede ser caracterizado de manera objetiva, que puede ser objeto de conocimiento teórico: para la teoría del significado literal, una interpretación sería correcta cuando coincida con el significado que de hecho se da a las palabras, mientras que para la teoría intencionalista, una interpretación sería correcta cuando coincida con la intención que de hecho tenía el legislador (suponiendo la existencia y posibilidad de conocimiento de dicha intención $\left.{ }^{20}\right)$. En mi opinión ninguna de esas dos

19 Cfr. Guastini, 2008, pp. 165-174.

${ }^{20}$ Sobre los problemas de una concepción de la interpretación en estos términos me he ocupado con detenimiento en un trabajo anterior (Lifante Vidal, 1999). 
teorías resulta adecuada para reconstruir por sí sola los aspectos normativos de la práctica interpretativa jurídica ${ }^{21}$. Ambas estarían tratando un problema práctico (cómo debe interpretarse "D") como si se tratase de una cuestión teórica, incurriendo así en otro de los clásicos paralogismos señalados por Vaz Ferreira; este paralogismo implica no tener en cuenta que el término "solución" no significa lo mismo en un ámbito teórico (donde se considera que hay soluciones exactas y unívocas que pueden ser conocidas objetivamente) que en un ámbito práctico, donde encontrar una "solución" suele implicar llevar a cabo valoraciones y ponderar los pros y contras de las distintas posibilidades, etc. Ser conscientes de ello no implica negar que alguna "solución" pueda resultar mejor que las otras, y que en este sentido debe ser preferida (aunque la misma no resulte "perfecta" o "correcta" en el mismo sentido que puede serlo una solución a un problema matemático).

Pero hemos de ser conscientes de que esas dos teorías no agotan todas las posibilidades de reconstrucción de los aspectos normativos de la práctica interpretativa. Podemos considerar que los criterios de corrección de la interpretación en el ámbito jurídico no vienen dados por su correspondencia exacta con un hecho "objetivo", sino que la mejor interpretación en el ámbito jurídico (la que debe ser por tanto preferida) será aquella que desarrolle al máximo los valores que la práctica jurídica, el Derecho, pretende desarrollar. Pero obviamente ésta es una caracterización "constructiva" o "valorativa" 22 de la actividad interpretativa; una caracterización que en mi opinión reconstruye mejor

${ }^{21}$ El significado literal (y quizás también en algunos casos la intención del legislador) juega un papel relevante en la interpretación jurídica; es el punto de partida, y en algunas ocasiones también puede ser el de llegada, pero el proceso interpretativo implica atender a razones de por qué interpretar en un cierto sentido que necesariamente no pueden ser el mero dato objetivo de ese significado literal (o de esa intención). Cfr. MacCormick, 1993.

${ }^{22} \mathrm{Y}$, como el lector habrá podido adivinar, muy similar a la caracterización que realizaría Dworkin, aunque la misma no tiene por qué comprometerse-como hace el autor norteamericano- con la existencia de una única respuesta correcta para todos -o casi todos- los casos difíciles. 
que la guastiniana cómo es en realidad la práctica interpretativa. Guastini podría aquí objetar que una teoría de este tipo en realidad no es en absoluto una "teoría", pues pretende decirnos cómo se debe interpretar y por lo tanto se ubicaría en un ámbito no descriptivo sino normativo. Pero lo que he intentado mostrar es que ese presupuesto metodológico del que parte Guastini, el "segregacionismo discursivo", según el cual se debe fragmentar la experiencia jurídica en discursos desconectados entre sí: por un lado un discurso teórico o descriptivo de hechos objetivos y susceptible de verificabilidad empírica, y por otro lado, un discurso puramente prescriptivo, reducido al ámbito de la subjetividad, y alejado de cualquier pretensión de racionalidad, es un presupuesto claramente inadecuado si lo que pretendemos es "reconstruir" la práctica interpretativa jurídica.

\section{Conclusiones}

Como señalaba al principio de este trabajo, mis observaciones no pretenden afectar a la utilidad analítica de las distinciones y contraposiciones guastinianas como modo de ordenar el discurso, sino a los riesgos que el abuso de las mismas entraña. Y en este sentido he intentado mostrar que el propio Guastini no puede sortear esos riesgos y acaba incurriendo en algunos paralogismos que le impiden ofrecer una caracterización adecuada de en qué consiste la actividad interpretativa en el ámbito jurídico. La caracterización de Guastini se sintetiza en lo que he llamado la tesis del "escepticismo moderado", que se compone a su vez de dos partes: por un lado, la tesis escéptica (toda disposición admite más de un significado junto a la inexistencia de valor de verdad o corrección en el ámbito de la interpretación) y por otro lado, la tesis moderada (la existencia de significados previos a la interpretación que limitan la actividad interpretativa). Pero los argumentos en los que Guastini basa su escepticismo resultan incompatibles con la afirmación de que existen límites a la actividad interpretativa, de manera que sus tesis escépticas acaban conduciendo a la tesis de que todo vale en materia de interpretación (el intérprete sería, entonces, el único y verdadero creador del Derecho), tesis de la que -con muy buen criterio- 
Guastini pretende apartarse. La imposibilidad de presentar una caracterización "adecuada" de la interpretación jurídica (una caracterización que no desemboque en ese escepticismo radical) se debe a que el presupuesto metodológico que opera como punto de partida del trabajo de Guastini es el segregacionismo discursivo, que le hace rechazar la posibilidad de explorar una caracterización "constructiva" de la interpretación jurídica como actividad no meramente descriptiva (objetiva) ni meramente prescriptiva (subjetiva). Según esta "tercera vía", la interpretación sería una actividad "participativa" (en la práctica), una actividad que requiere llevar a cabo valoraciones y que incorpora criterios de corrección.

\section{Bibliografía}

Aguiló Regla, Josep (2011): "El constitucionalismo imposible de Luigi Ferrajoli", en Doxa, 34.

Alchourrón, Carlos y Bulygin, Eugenio (1987): Introducción a la metodología de las ciencias jurídicas y sociales, Astrea, Buenos Aires.

BARBERIS, Mauro (2000): "Lo scetticismo immaginario. Nove obiezioni agli scettici à la génoise", en Analisi e diritto, 2000, pp. 1-37.

Chiassoni, Pierluigi (1998): "L'ineluttabile scetticismo della 'scuola genovese", en Analisi e diritto, 1998, pp. 21-76.

Dworkin, Ronad (1986): Law's Empire, en Belknap Press, Harvard.

GuAstinI, Riccardo, (1999): Distinguiendo. Estudios de teoría y metateoría del Derecho, trad. J. Ferrer, Gedisa, Barcelona.

GuAstinI, Riccardo (2008): Nuovi studi sull'interpretazione, Aracne, Roma.

GuAstini, Riccardo, (2010), "Fundamentos de una teoría escéptica de la interpretación”, trad. D. Moreno Cruz, en I. Lifante Vidal (ed.), Interpretación jurídica y teoría del Derecho, Palestra, pp. 147-172.

LifANTE VIDAL, Isabel (1999): "Interpretación y modelos de Derecho. Sobre el papel de la intención en la interpretación jurídica”, en Doxa. Cuadernos de Filosofia del Derecho, nº 22, 1999, págs. 171-193. 
Distinciones y paralogismos. A propósito...

LIFANTE VIDAL, Isabel (2010): "Un mapa de problemas sobre la interpretación jurídica", en I. Lifante Vidal (ed.), Interpretación jurídica y teoría del Derecho, Palestra, pp. 37-64.

MACCORMick, Neil (1978): Legal Reasoning and Legal Theory, Clarendon Press, Oxford.

MACCORMICK, Neil (1993): "Argumentation and Interpretation in Law", en Ratio Iuris, marzo, 1993, pp. 16-29.

MARTÍNEZ, Sergio F. (2008): "Un lugar para las prácticas en una filosofía de la ciencia", en J. Miguel Esteban y Sergio F. Martínez (eds.), Normas y prácticas en la ciencia, Instituto de Investigaciones Filosóficas, UNAM, México, pp. 151-167.

Ross, Alf (1970): Sobre el Derecho y la justicia, trad. G. Carrió, Eudeba, Buenos Aires.

Rouse, Joseph (2008): "Dos conceptos de práctica", trad. J. García Campos, en J. Miguel Esteban y Sergio F. Martínez (eds.), Normas y prácticas en la ciencia, Instituto de Investigaciones Filosóficas, UNAM, México, pp. 19-34.

TARELLO, Giovanni (1980): L'interpretazione della legge, Giuffrè, Milano. Vaz Ferreira, Carlos (1979): "Lógica viva”, en Lógica viva. Moral para intelectuales, Biblioteca Ayacucho, Venezuela, pp. 3-190.

VeGA ReÑon, Luis (2008): "Paralogismos. Una contribución de C. Vaz Ferreira al análisis de la argumentación falaz", en Doxa, 31, pp. 625640. 